\title{
Das weltweite WHO-MONICA-Projekt: Ergebnisse und Ausblick
}

The Worldwide WHO MONICA Project: Results and Perspectives

\section{Zusammenfassung}

Nach dem 2. Weltkrieg hat die koronare Herzkrankheit (KHK) in den westlichen Ländern epidemische Ausmaße angenommen. Der Höhepunkt der Epidemie wurde in vielen Ländern 1968 erreicht. 1978 fand in Bethesda eine Konferenz der National Institutes of Health mit dem Titel „The Decline in CHD Mortality“ statt. Bei dieser Konferenz sollte geklärt werden, ob präventivmedizinische Programme oder Verbesserungen der Akutbehandlung zum Rückgang der altersspezifischen Mortalitätsraten an KHK geführt hatten. Aufgrund der mangelhaften Datenlage konnte diese Frage 1978 nicht beantwortet werden. Das WHOMONICA-Projekt griff diese Frage auf und wurde als weltweites Monitoring-Projekt für die Erfassung von Trends und Determinanten der Herz-Kreislauf-Mortalität und -Morbidität konzipiert und von Mitte der 80er- bis Mitte der 90er-Jahre in 38 Populationen in 21 Ländern der Erde durchgeführt. Insgesamt standen 13 Millionen Menschen über 10 Jahre unter Beobachtung. 166000 Herzinfarktpatienten wurden registriert und über 300000 Menschen wurden auf Stichprobenbasis auf ihr kardiovaskuläres Risikofaktorenprofil hin untersucht. In den westlichen Ländern, in denen die Herzinfarktmortalität durchschnittlich um 2-3\% jährlich abgenommen hatte, konnten zwei Drittel des Rückgangs auf eine Verminderung der Inzidenz und ein Drittel auf eine Verminderung der Letalität zurückgeführt werden. Wenn man die Daten aller MONICA-Populationen betrachtet, sieht man, dass der größte Beitrag zum Rückgang der Herzinfarktmortalität bei Männern vom Rückgang des Rauchens herrührt. Neben der Sieben-Länder-Studie und der Framingham-Studie kommt dem WHO-MONICA-Projekt die größte Bedeutung für die Weiterentwicklung der Herz-Kreislauf-Epidemiologie und Prävention in der Welt zu.

\section{Abstract}

After World War II coronary heart disease (CHD) assumed epidemic proportions in western countries. In many countries the peak of the epidemic occurred in 1968. In 1978 the National Heart, Lung and Blood Institute of the NIH organized the Bethesda conference on the decline in CHD mortality. The aim of the conference was to find out whether measures of prevention or improvements in acute coronary care were responsible for the decline in age-specific CHD mortality rates. Because of lack of appropriate data in 1978 these questions remained unanswered. To answer these questions the WHO MONICA (Monitoring trends and determinants in cardiovascular disease) project was organized as a monitoring system to assess trends and determinants of cardiovascular mortality, incidence and case fatality from the mid $1980 \mathrm{~s}$ to the mid $1990 \mathrm{~s}$ in 38 populations in 21 countries worldwide. Altogether some 13 million people were monitored over a 10 year period. 166,000 myocardial infarction patients were registered and more than 300,000 men and women were sampled and examined for their cardiovascular risk factors and many other health data. In Western countries, where the CHD mortality decline was on average $2-3 \%$ annually, two thirds of this decline could be explained by a decline in CHD incidence and one third by a decline in CHD case fatality. When relating risk factor changes to changes in CHD event rates in men over a time period of 10 years in all MONICA populations it turned out that the greatest contribution to the CHD decline came from a decrease in smoking. On a worldwide scale the Seven Countries Study, the Framingham Heart Study and the WHO MONICA Project have contributed most to the development of epidemiology and prevention of cardiovascular diseases.

Institutsangaben

Universität Münster, Institut für Epidemiologie und Sozialmedizin, Münster

Korrespondenzadresse

Univ.-Prof. Dr. med. Ulrich Keil, Ph. D. · Institut für Epidemiologie und Sozialmedizin · Universität Münster UKM · Domagkstraße 3 · 48149 Münster · E-mail: keilu@uni-muenster.de

Bibliografie

Gesundheitswesen 2005; 67 Sonderheft 1: S38 -S45 @ Georg Thieme Verlag KG Stuttgart • New York DOI $10.1055 / \mathrm{s}-2005-858240$

ISSN 0949-7013 


\section{Einleitung}

Nach dem 2. Weltkrieg hat die koronare Herzkrankheit (KHK, Herzinfarkt) in den westlichen Ländern epidemische Ausmaße angenommen. In den USA und anderen westlichen Ländern wie z.B. Australien und Kanada wurde der Höhepunkt der Epidemie 1968 erreicht. 1978 fand in Bethesda bei Washington eine Konferenz der National Institutes of Health unter dem Titel „The Decline in Coronary Heart Disease Mortality“ statt [1]. Bei dieser Konferenz sollte geklärt werden, welche Faktoren für den Rückgang der KHK-Epidemie verantwortlich gemacht werden konnten. Waren es die präventivmedizinischen Strategien und Programme, oder konnte man den Rückgang der altersspezifischen Mortalitätsraten an KHK auf Verbesserungen der Behandlung von KHK-Patienten zurückführen? (Abb.1) Es ging also um die Frage, ob der Rückgang der Herzinfarktmortalität durch einen Rückgang der Inzidenz (Neuerkrankungsziffer) oder durch eine Verminderung der Letalität (Sterblichkeit nach Eintreten des Infarktes) oder durch beide zu erklären war. Aufgrund der mangelhaften Datenlage im Jahr 1978 konnte man diese eminent wichtige Frage damals nicht beantworten. Zudem wurde von einigen Experten bezweifelt, ob die Qualität der offiziellen Mortalitätsdaten zur Messung der Trends kardiovaskulärer Erkrankungen ausreichen würde. Vor der Erklärung der Trends musste deshalb zunächst einmal die Validität der kardiovaskulären Mortalitätsdaten geklärt werden.

\section{Methodik}

Das MONICA-Projekt der Weltgesundheitsorganisation (MONI$\mathrm{CA}=$ Monitoring Trends and Determinants in Cardiovascular Disease) griff diese Frage auf und wurde als weltweites Monito-

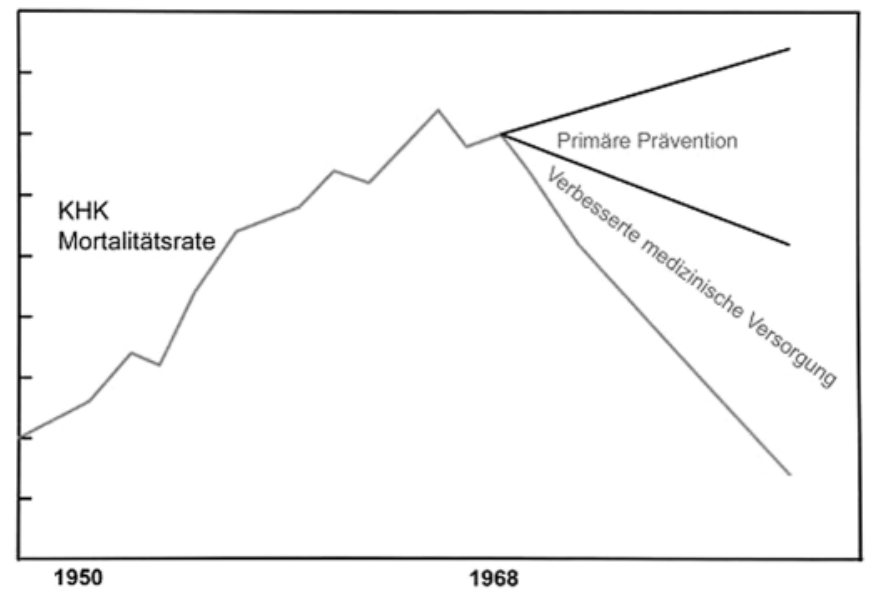

Abb. 1 Können wir die Faktoren identifizieren, die für den Rückgang der Mortalität an koronarer Herzkrankheit (KHK) verantwortlich sind? KHK-Mortalitätsrate in Abhängigkeit von primärer Prävention und/ oder verbesserter medizinischer Versorgung [1].
ring-Projekt für die Validierung der offiziellen Mortalitätsdaten für Herz-Kreislauf-Erkrankungen und für die Erfassung von Trends und Determinanten der Herz-Kreislauf-Mortalität und -Morbidität konzipiert und von Mitte der 80er- bis Mitte der 90er-Jahre in 38 Populationen (Altersgruppe 35-64 Jahre) in 21 Ländern der Erde durchgeführt [2]. Die 38 Populationen verteilten sich auf Europa, Asien, Australien, Neuseeland und Nordamerika. Wegen der hohen Ansprüche an die Datenqualität konnten leider keine MONICA-Zentren in Südamerika, Afrika oder Indien eingerichtet werden. Insgesamt standen in den 38 Populationen über 13 Millionen Menschen über 10 Jahre unter Beobachtung. 166000 Herzinfarktpatienten wurden registriert, und über 300000 Menschen wurden auf Stichprobenbasis auf ihr kardiovaskuläres Risikofaktorenprofil hin untersucht. Deutschland war mit den Regionen Augsburg, Bremen, Chemnitz, Erfurt und Zwickau an diesem weltweit größten medizinischen Forschungsprojekt beteiligt [2].

Die MONICA-Weltkarte (Abb. 2) zeigt die Verteilung der MONICAZentren von Auckland/Neuseeland bis Stanford in Kalifornien, wobei deutlich wird, dass sich der Schwerpunkt der MONICA-Zentren in Europa befindet (Abb.3). Die für das internationale MONICAProjekt notwendigen Daten wurden in den 38 genau definierten Populationen an Personen der Altersgruppe 25-64 erhoben. Vier Gruppen von Daten sind zu unterscheiden:

1. demografische Daten und Mortalitätsdaten pro Jahr,

2. Daten aus drei Querschnittsstudien,

3. Inzidenzraten für Herzinfarkt und Schlaganfall (Registerdaten)

4. und Daten über die medizinische Versorgung.

Die demografischen Daten sind für die Berechnung von Inzidenz-, Prävalenz- und Mortalitätsraten unentbehrlich, weil sie bei der Berechnung dieser Raten die Bezugshäufigkeit, also den Nenner bilden.

Für die drei Querschnittsstudien wurden voneinander unabhängige Zufallsstichproben am Anfang, in der Mitte und am Ende der 10-Jahres-Periode gezogen. Mit diesen Daten waren Schätzungen des kardiovaskulären Risikofaktorenprofils und seiner Veränderungen über 10 Jahre möglich. Die bevölkerungsbasierten Herzinfarkt-Registerdaten ließen Aussagen über den Verlauf der Mortalität, der Inzidenz und der Letalität an KHK zu. Die Daten über die medizinische Versorgung erlaubten Aussagen über deren Einfluss auf Mortalität und Letalität an kardiovaskulären Krankheiten. Das für alle MONICA-Zentren geltende Studiendesign geht aus Abb. 4 (MONICA Augsburg) hervor.

Um sicherzustellen, dass in jedem einzelnen MONICA-Zentrum die Trends der koronaren Mortalität und Morbidität und die Trends der klassischen Risikofaktoren (Rauchen, Hypertonie, Hypercholesterinämie) mit genügender Genauigkeit gemessen werden konnten, wurden Stichprobenberechnungen mit verschiedenen Annahmen durchgeführt [2]. Man kam überein, dass 


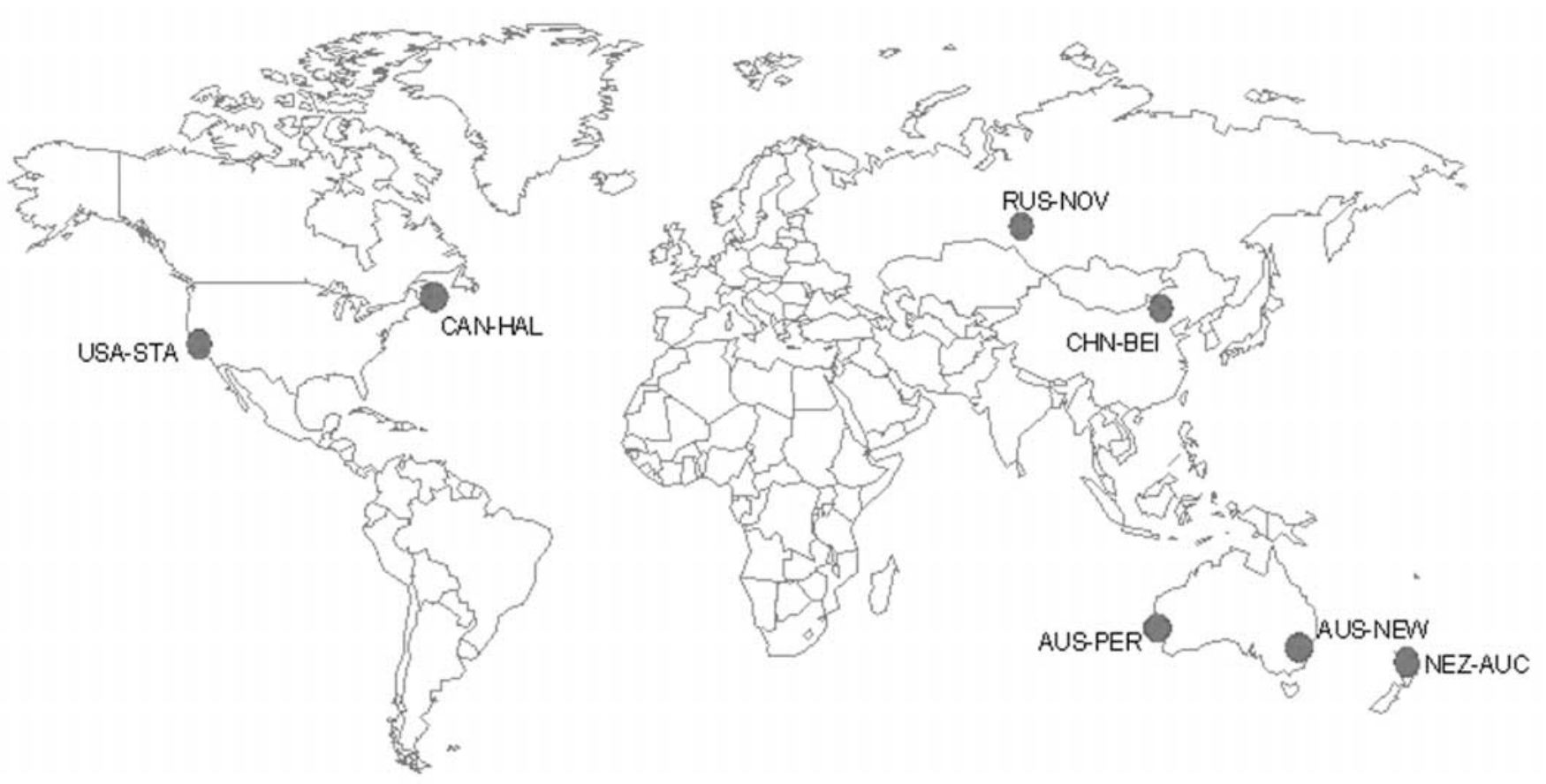

Abb. 2 Weltkarte der außereuropäischen WHO-MONICA-Zentren.

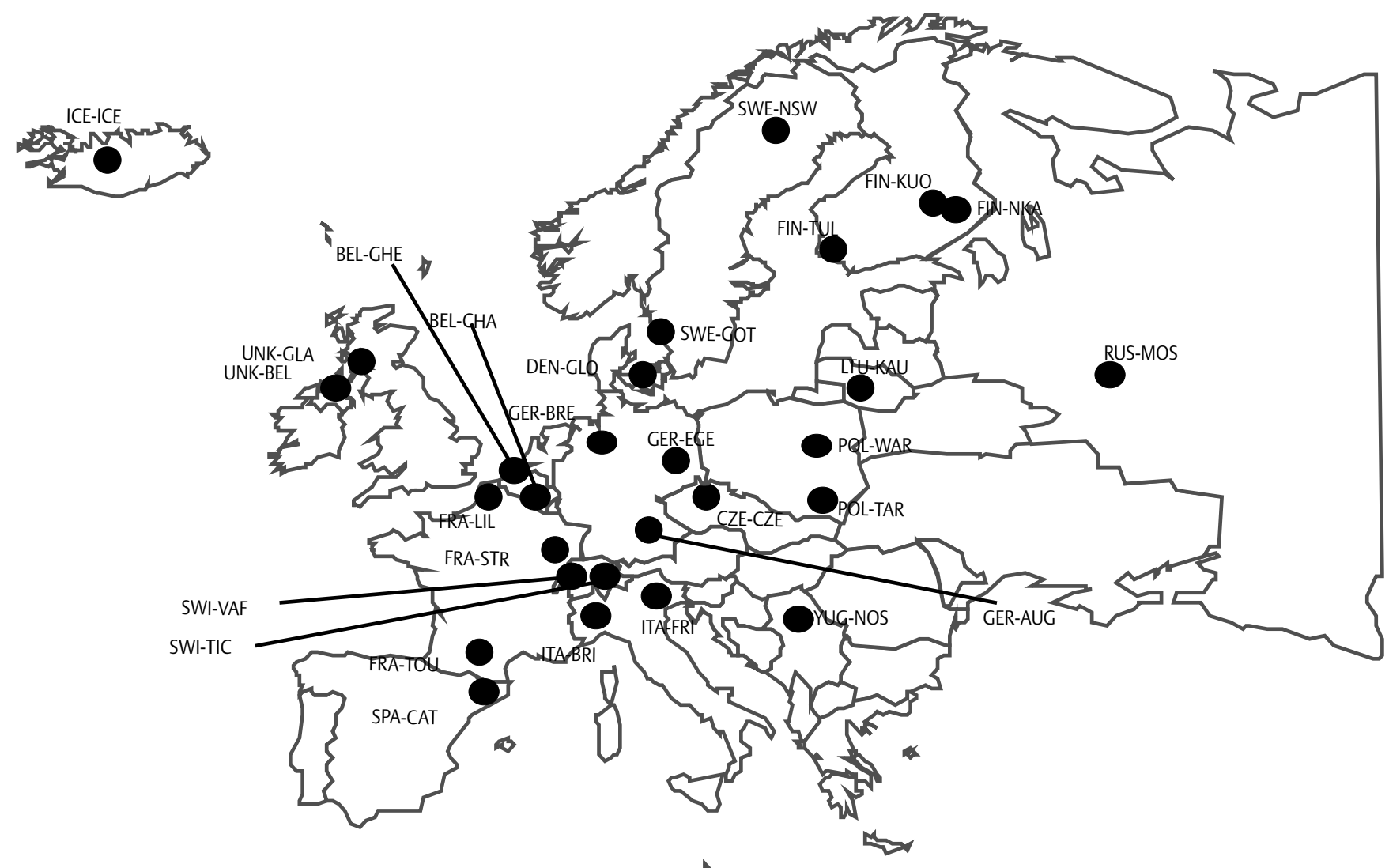

Quelle: Tunstall-Pedoe H (ed.) MONICA monograph and multimedia sourcebook. 2003, p. 159

Abb. 3 Europäische WHO-MONICA-Zentren.

in jedem einzelnen MONICA-Zentrum mindestens 200 tödliche KHK-Ereignisse pro Jahr bei Männern in der Altersgruppe 25 - 64 auftreten müssten, um genügend genaue Trendanalysen pro Zentrum vornehmen zu können. Die Stichprobenberechnungen für die einzelnen Querschnittsstudien zur Erfassung der kardiovaskulären Risikofaktoren ergaben, dass für jede 10-Jahres-Altersgruppe und getrennt für jedes Geschlecht je 200 Personen ausgewählt werden mussten. Dies ergab für Männer und Frauen 


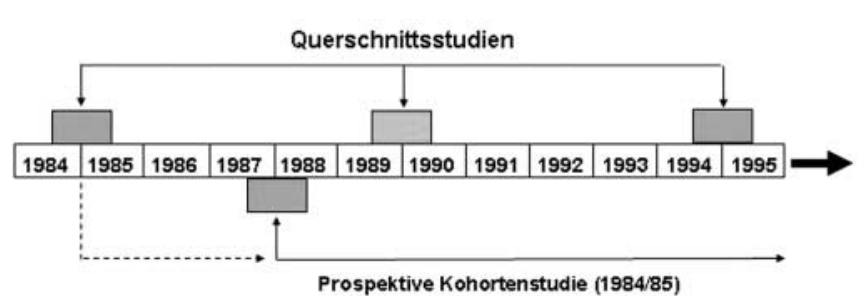

1.10 .1984

Bevolkerungsbasiertes Herzinfarktregister mit Follow-Up der Patienten

\begin{tabular}{|l|l|l|l|l|l|l|l|l|l|l|l|}
\hline 1984 & 1985 & 1986 & 1987 & 1988 & 1989 & 1990 & 1991 & 1992 & 1993 & 1994 & 1995 \\
\hline
\end{tabular}

Abb. 4 Studiendesign und Zeitplan

(MONICA Augsburg als Beispiel).

der Altersgruppe 25 - 64 Jahre insgesamt 1600 Personen [2]. Die meisten MONICA-Zentren wählten aber für ihre repräsentativen Querschnittsstudien weit größere Stichproben aus.

Da das MONICA-Projekt in 38 Zentren weltweit durchgeführt wurde, war die Standardisierung der Datenerhebung - innerhalb eines Zentrums und zwischen den Zentren - und die Beibehaltung einer hohen Datenqualität in allen Zentren über mindestens 10 Jahre eine große organisatorische Herausforderung [2]. Um eine hohe Datenqualität zu gewährleisten, wurden Qualitätszentren für Lipidbestimmungen, für EKG-Kodierungen und für Herz-
Qualitätszentren zusammen mit einem Koordinationszentrum und einem Datenzentrum führten die Hauptaktivitäten des Gesamtprojektes durch. Sie arbeiteten über mehr als 10 Jahre unter Aufsicht des MONICA-Parlamentes (38 Mitglieder) und des von ihm gewählten Leitbüros (Steering Committees - 6 Mitglieder). Die Organisationsstruktur des gesamten MONICA-Projektes geht aus Abb. 5 hervor [2].

Die wichtigste Arbeit fiel zweifellos jedem einzelnen MONICAZentrum zu, welches die Daten im jeweiligen Studiengebiet erhob und lokal auswertete. Dem MONICA-Datenzentrum in Helsinki kam die Aufgabe zu, die Daten aller 38 Zentren gemeinsam zu analysieren und aufgrund der gepoolten Daten aller Zentren die Trends der kardiovaskulären Mortalität und Morbidität mithilfe der Veränderung der klassischen Risikofaktoren und den Veränderungen der Akutversorgung zu erklären.

\section{Ergebnisse}

Die Validierung der offiziellen Mortalitätsdaten zeigte, dass in den verschiedenen MONICA-Zentren unterschiedliche Kodierungen für KHK vorgenommen wurden. Deshalb sind die offiziellen Mortalitätsdaten für KHK für internationale Vergleiche nur bedingt geeignet [3]. Für Trendanalysen pro Land eignen sich aber die offiziellen KHK-Mortalitätsdaten in vielen Ländern. In den westlichen Ländern, in denen die Herzinfarktmortalität um 2

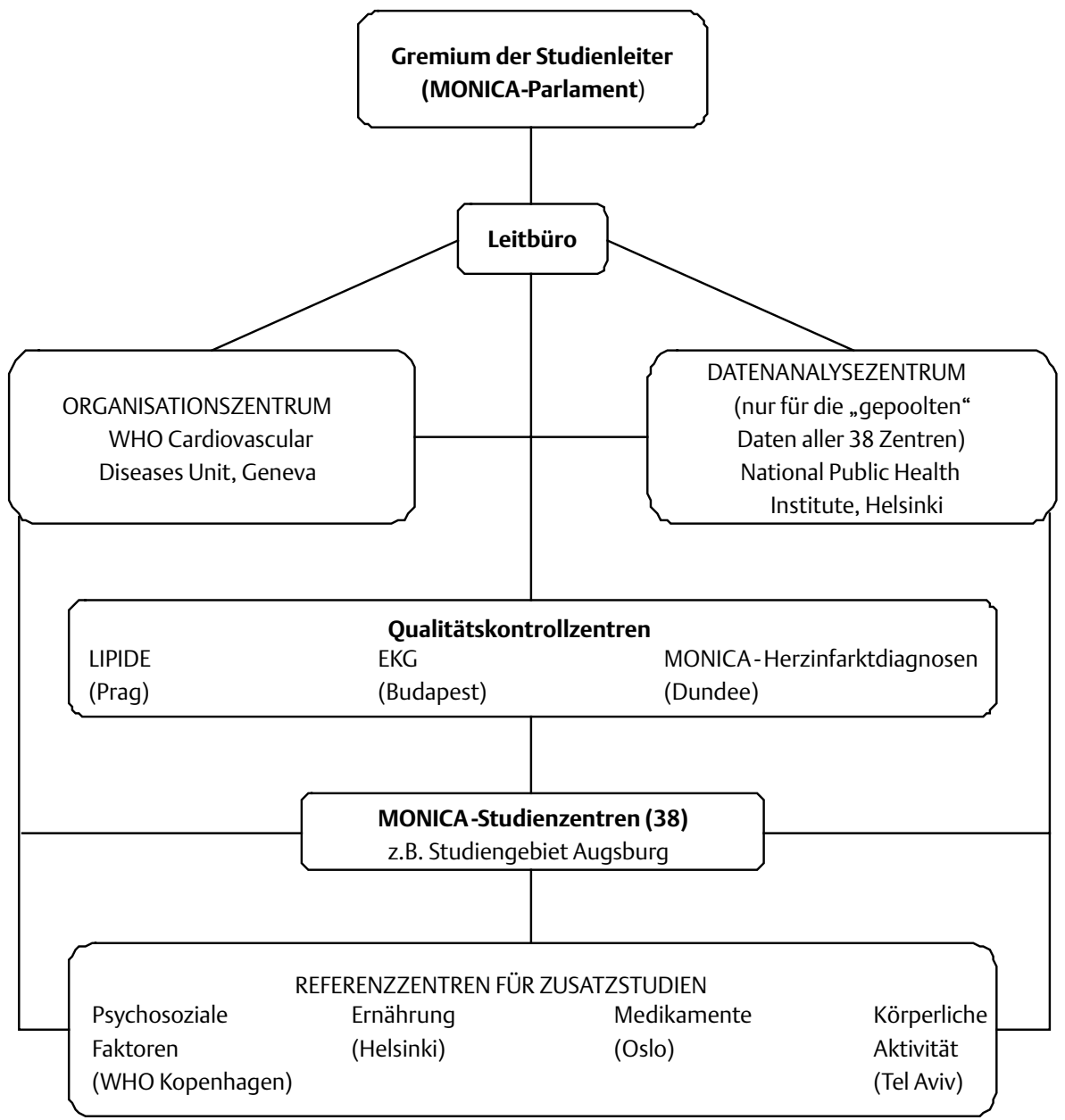

Abb. 5 Das MONICA-Projekt der Weltgesundheitsorganisation (WHO); Organisationsstruktur [2]. 

20 bis 30\%, konnten zwei Drittel des Rückganges auf eine Verminderung der Inzidenz und ein Drittel auf eine Verminderung der Letalität zurückgeführt werden $[3,4]$.

Bei der Konzeption des MONICA-Projektes Ende der 70er- und Anfang der 80er-Jahre des vergangenen Jahrhunderts hatte man angenommen, dass ein Rückgang der Inzidenz auf eine Verminderung der klassischen Risikofaktoren Rauchen, hohe Cholesterinspiegel und hohe Blutdruckwerte zurückzuführen sei, während die Letalität, d.h. die Sterblichkeit nach Herzinfarkt, oder positiv ausgedrückt die Überlebenswahrscheinlichkeit nach Infarkt in engem Zusammenhang mit den Fortschritten der medizinischen Akutversorgung stehen sollte.

Die in der Lancet-Ausgabe vom Mai 1999 [3] veröffentlichten Daten können so interpretiert werden, dass präventivmedizinische Maßnahmen am Rückgang der altersspezifischen Herzinfarktmortalität mit etwa zwei Dritteln und die Verbesserung der Akutversorgung mit einem Drittel beteiligt sind. Noch vor Beginn des neuen Millenniums konnte also die 1978 in Bethesda formulierte Frage nach der Bedeutung der Prävention bzw. der Akutversorgung für den Rückgang der KHK-Mortalität mit Daten des internationalen MONICA-Projektes beantwortet werden [3, 4] (Abb. 6).

Am 26. Februar 2000 sind zwei weitere Artikel im Lancet mit Schlussergebnissen des weltweiten Projektes erschienen [6, 7]. Sie gehen spezifischer auf die Bedeutung der Veränderungen

\section{Männer (35-64 Jahre)}

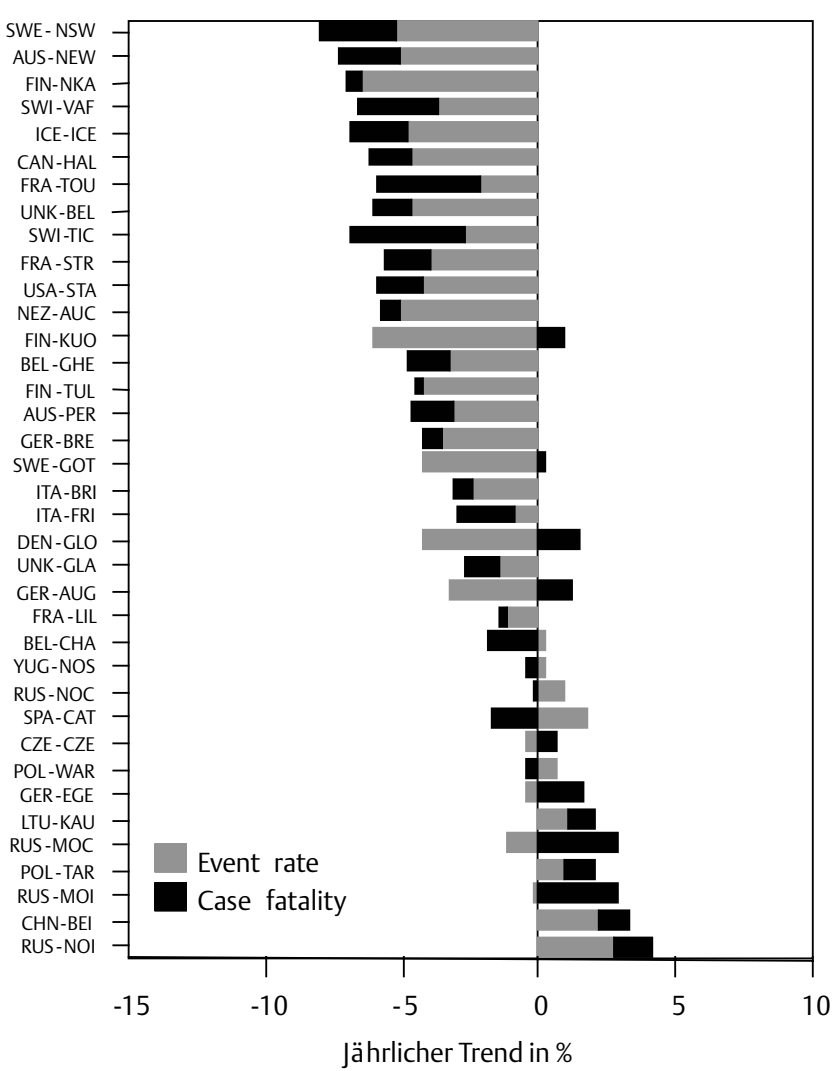

der klassischen Risikofaktoren auf die KHK-Ereignisrate und der akuten Koronarversorgung auf Morbidität, Mortalität und Letalität an KHK ein.

Bei den untersuchten Risikofaktoren handelt es sich um Zigarettenrauchen, Blutdruck, Cholesterin und Körpergewicht. Bei der Untersuchung der Behandlung ging es um evidenzbasierte Verfahren, wie Medikation mit Aspirin, Betablockern, ACE-Inhibitoren, Bypass-Operation/Balondilatation und Thrombolyse. Von den genannten Risikofaktoren und Therapieverfahren wissen wir seit geraumer Zeit aus epidemiologischen Kohortenstudien und aus randomisierten kontrollierten Studien (Clinical Trials), dass sie für Erkrankungsrisiko und Überleben nach Infarkt beim einzelnen Patienten von großer Bedeutung sind. Die große Herausforderung für das WHO-MONICA-Projekt bestand darin herauszufinden, welchen Einfluss die Veränderungen der Risikofaktoren und die Veränderungen der Therapieverfahren auf die Trends der Herzinfarktraten in ganzen Populationen hatten. Es ist offensichtlich, dass diese Frage für Medizin und Gesundheitspolitik von großer Bedeutung ist [5].

\section{Ergebnisse hinsichtlich Risikofaktoren}

Bei den Männern nahmen die Risikofaktoren Zigarettenrauchen, hoher Blutdruck und hohe Cholesterinspiegel zumindest in den MONICA-Zentren in westlichen Ländern ab, während bei Frauen das Rauchen in den verschiedenen Ländern unterschiedliche

Frauen (35-64Jahre)

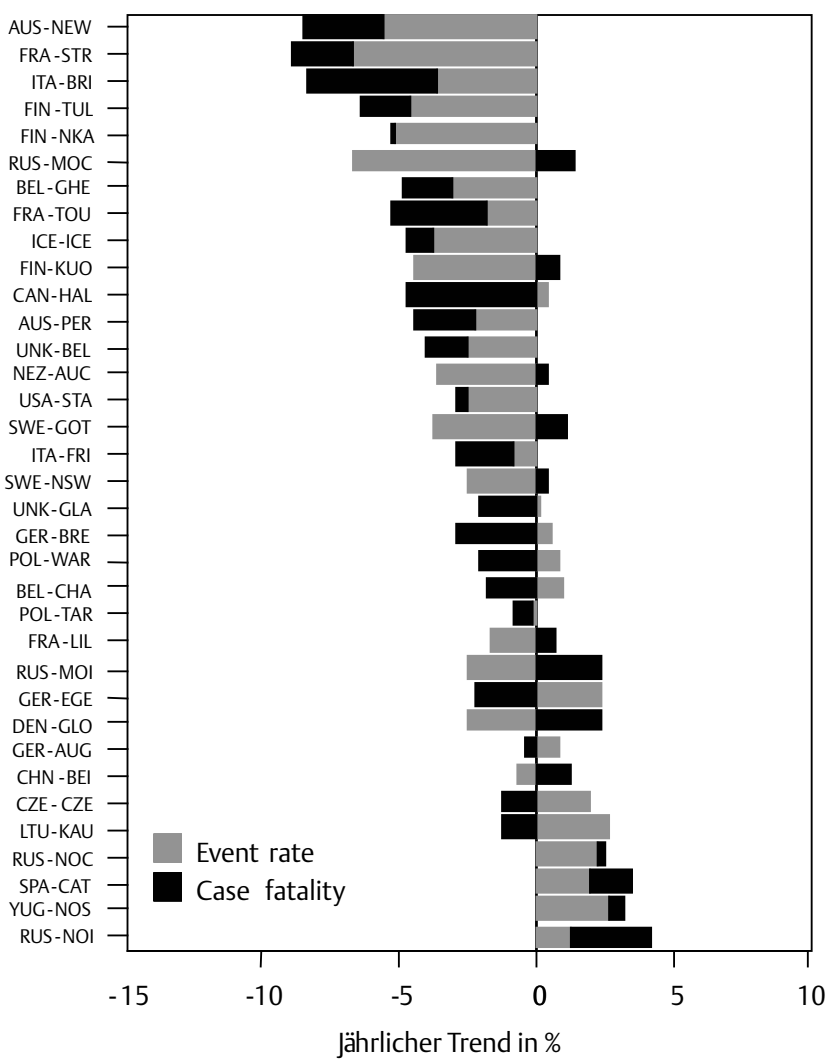

Quelle: Tunstall-Pedoe H (ed.) MONICA monograph and multimedia sourcebook. 2003, p. 181

Abb. 6 Veränderungen der KHK-Mortalitätsraten (nach MONICA-Kriterien) stratifiziert nach Veränderungen der KHK-Ereignisraten und nach Veränderungen der KHK-Letalität (case fatality) [3, 5]. 


\begin{tabular}{|c|c|c|c|c|}
\hline \multicolumn{2}{|l|}{ KHK-Ereignisse } & \multicolumn{2}{|c|}{ Koeffizient (95\% KI) } & \multirow[t]{2}{*}{$\begin{array}{l}\text { Prozent der erklärten } \\
\text { Variabilität }\end{array}$} \\
\hline \multicolumn{4}{|l|}{ KHK- Ereignisse } & \\
\hline \multicolumn{5}{|c|}{ Zeitversetzte Registrierung } \\
\hline \multirow[t]{3}{*}{ Einfache Regression ${ }^{1}$} & Rauchen (\%) & 0,019 & $(0,00$ bis 0,04$)$ & 20 \\
\hline & $\mathrm{SBD}(\mathrm{mm} \mathrm{Hg})$ & 0,008 & $(-0,01$ bis 0,03$)$ & 6 \\
\hline & $\mathrm{BMI}\left(\mathrm{kg} / \mathrm{m}^{2}\right)$ & $-0,23$ & $(-0,39$ bis $-0,07$ & 36 \\
\hline \multirow[t]{3}{*}{ Multiple Regression ${ }^{1}$} & Rauchen (\%) & 0,009 & $(-0,01$ bis 0,03$)$ & 46 \\
\hline & $\mathrm{SBD}(\mathrm{mm} \mathrm{Hg})$ & 0,007 & $(-0,02$ bis 0,03$)$ & \\
\hline & Gesamt-Chol. (mmol/L) & 0,19 & $(-0,05$ bis 0,42$)$ & \\
\hline
\end{tabular}

Regression der zeitlichen Trends der KHK-Ereignisraten gemäß Trends der einzelnen Risikofaktoren bei 35- bis 64-jährigen Männern [6]. KHK = koronare Herzkrankheit, $\mathrm{SBD}=$ systolischer Blutdruck, BMI = Body Mass Index, GesamtChol. $=$ Gesamtcholes terin

${ }^{1}$ Gewichtet nach Qualitäts-Score [6].

Trends aufwies. Dagegen nahm das Gewicht bei Männern und Frauen in den meisten MONICA-Regionen zu [5, 6].

Wenn man die Daten aller Populationen betrachtet, sieht man, dass der größte Beitrag zum Rückgang der altersspezifischen Herzinfarktmortalität bei Männern, wie er in nahezu allen westlichen Ländern beobachtet wurde, vom Rückgang des Rauchens herrührt, während entsprechende Veränderungen der Herzinfarktmortalität bei Frauen stärker auf fallende Blutdruckwerte bzw. bessere Behandlung des Blutdrucks zurückzuführen sind. Die Beziehungen zwischen dem Rückgang der Herzinfarktraten und den Veränderungen der klassischen Risikofaktoren waren nicht so stark, wie man das von individuellen epidemiologischen Kohortenstudien und den Clinical Trials her erwartet hätte. Die Beziehungen waren bei Männern stärker als bei Frauen, und sie wurden verstärkt, wenn eine Latenzzeitperiode zwischen Risikofaktorenveränderung und Auftreten von Herzinfarkt in die Analysen eingeführt wurde [6].

Tab. 1 zeigt für Männer der Altersgruppe 35-64 Jahre, dass durch die Risikofaktorenveränderungen rund $46 \%$ der KHK-Variabilität erklärt werden können (multiple Regressionsberechnung). Dieses Ergebnis kontrastiert mit Aussagen aus prospektiven Kohortenstudien, aus denen hervorgeht, dass mit den drei klassischen Risikofaktoren etwa $80 \%$ der KHK-Variabilität erklärt werden können [7]. Die große Diskrepanz zwischen beiden Aussagen kann wie folgt erklärt werden: Bei der vorliegenden MONICA-Datenanalyse bewegen wir uns auf ökologischer bzw. Aggregatdatenebene. Auf dieser Ebene müssen wir mit einer Reihe von Fehlermöglichkeiten rechnen, die fast alle zu einer Abschwächung der Korrelation zwischen klassischen Risikofaktoren und KHK-Ereignisrate führen. Weiterhin sollten wir auch beachten, dass die Aussage, dass mit den drei klassischen Risikofaktoren etwa 80\% der KHK-Variabilität erklärt werden können, erst dann gilt, wenn in den prospektiven Kohortenstudien an Individuen für den „Regression Dilution Bias“ korrigiert wird [7]. Ohne Korrektur für den „Regression Dilution Bias" können etwa 65\% der KHK-Variabilität mit den klassischen Risikofaktoren erklärt werden [8].

Insgesamt unterstützt dieser Teil der MONICA-Aggregatdatenanalyse die Vorstellung von der Bedeutung der klassischen Risikofaktoren für das Auftreten der KHK. Es wird aus den Analysen aber auch deutlich, dass die relativ schwachen Korrelationen zwischen Veränderungen der klassischen Risikofaktoren und Veränderungen der KHK-Ereignisrate zum einen auf Schwierigkeiten bei der Messung der Risikofaktoren und der KHK-Ereignisse in diesem weltweiten Projekt zurückzuführen sind, zum anderen aber auch darauf, dass neben den klassischen Risikofaktoren natürlich auch Lebensstilfaktoren, wie körperliche Aktivität, Ernährungsweise, psychosoziale Faktoren und soziale Bedingungen für das Auftreten von Herzinfarkten von Bedeutung sind.

\section{Ergebnisse hinsichtlich Behandlung}

In den meisten MONICA-Populationen wurden im Beobachtungszeitraum von 10 Jahren starke Veränderungen bei den evidenzbasierten Therapieverfahren gesehen. Besonders dramatisch war die Zunahme der Behandlung mit Thrombozytenaggregationshemmern (Aspirin). Bei der Veränderung der Behandlung war auch ein starker Ost-West-Gradient zu beobachten, der mit entsprechendem Rückgang der KHK-Mortalität und -Morbidität im Westen und Stagnation bzw. Zunahme der KHK-Mortalität und -Morbidität in Osteuropa und besonders in Russland einherging. Wir sehen also eine starke Beziehung zwischen der Intensivierung der evidenzbasierten Therapie und dem Rückgang von KHK-Mortalität, -Morbidität und -Letalität [9].

Insgesamt kann man aus der gepoolten MONICA-Datenanalyse folgende Schlussfolgerungen für die Bevölkerung ziehen: Es lohnt sich, die klassischen Risikofaktoren Rauchen, hoher Blutdruck und hohes Cholesterin zu behandeln und zu kontrollieren, damit ein(e) Mann/Frau erst gar keine KHK entwickelt. Wer an KHK leidet oder ein hohes Risiko für KHK hat, sollte sich einer Behandlung mit den genannten evidenzbasierten Therapieverfahren unterziehen.

\section{Ausblick}

In Deutschland hat die koronare Herzkrankheit mit rund 300000 tödlichen und nichttödlichen Fällen im Jahr weiterhin epidemischen Charakter. Der primären und sekundären Prävention kommt deshalb größte Bedeutung zu. Für Deutschland wird dies besonders gut durch die MONICA-Daten der Region Augsburg belegt [10]: 
Männer

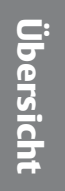

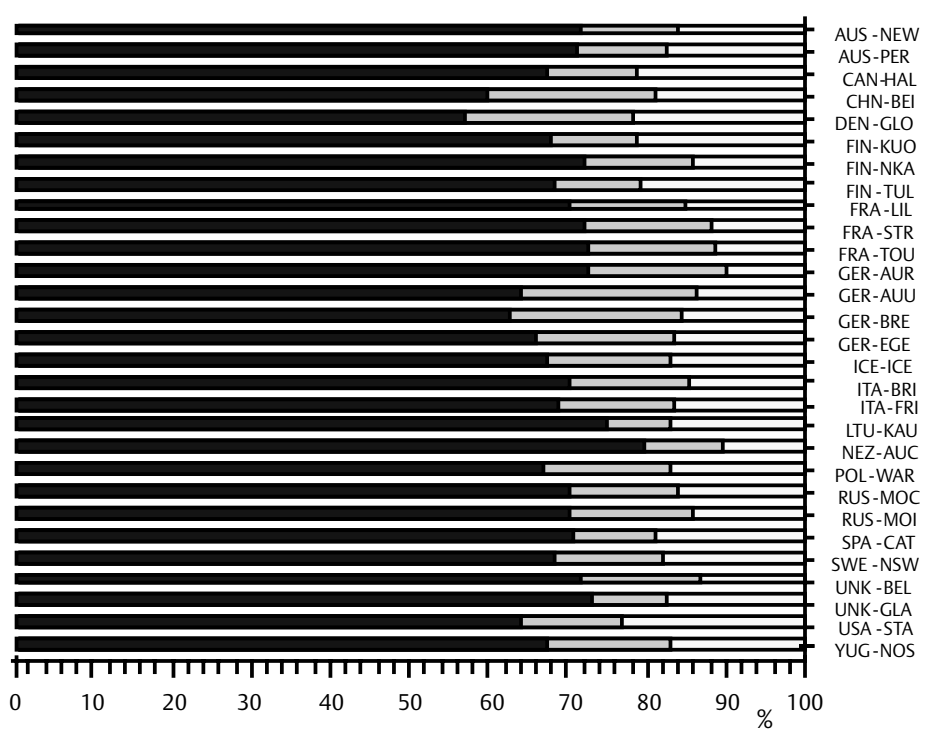

Frauen

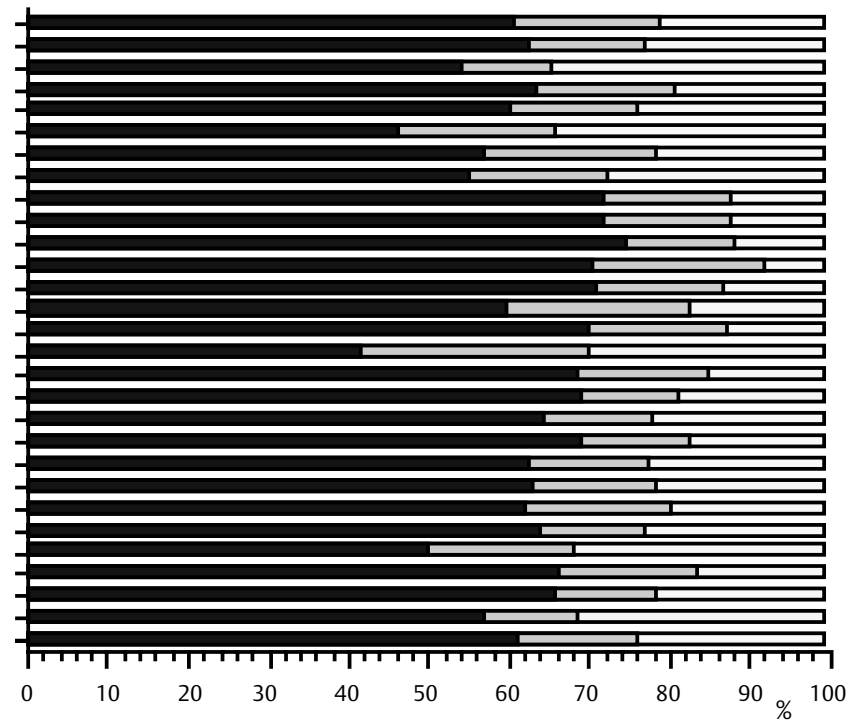

24 Stunden bis 28 Tage nach Beginn von Symptomen, für hospitalisierte Patienten

Innerhalb von 24 Stunden nach Beginn von Symptomen, für hospitalisierte Patienten

Quelle: Chambless L, Keil U, et al. Circulation 1997; 96: 3849-3859

Abb. 7 Prozentuale Verteilung der innerhalb von 28 Tagen tödlich verlaufenden KHK-Ereignisse nach Zeitpunkt des Todes: prähospital, für hospitalisierte Patienten innerhalb von 24 Stunden nach Beginn

1. $30 \%$ der Herzinfarktpatienten dieser Region sterben, bevor sie das Krankenhaus erreichen und moderne Therapieverfahren in Anspruch nehmen können.

2. Wenn man die Sterblichkeit an Herzinfarkt (Erst- und Reinfarkt) insgesamt betrachtet, d. h. die Sterblichkeit vor Krankenhausaufnahme und im Krankenhaus zusammen analysiert, kommt man auf den überraschend hohen Wert von 50\% [11].

3. Die Sterblichkeit bei Reinfarkt ist doppelt so hoch wie bei Erstinfarkt. Dies spricht besonders stark für die Notwendigkeit der Verbesserung der sekundären Prävention [11].

4. Von allen Patienten, die an Herzinfarkt versterben, versterben etwa $2 / 3$ außerhalb des Krankenhauses. An dieser Relation hat sich in den letzten 20 Jahren, trotz dramatischer Veränderungen der medizinischen Versorgung, wenig geändert [11] (Abb. 7).

Aus den bisherigen Ausführungen sollte nicht der vorschnelle Schluss gezogen werden, dass sich das internationale MONICAProjekt in den hier beschriebenen Hauptaussagen erschöpft. Die größte Bedeutung des WHO-MONICA-Projektes liegt vielmehr darin begründet, dass es vor mehr als 20 Jahren gelungen ist, ein weltweites Netzwerk von Herz-Kreislauf-Epidemiologen zu etablieren und Datenbasen zu schaffen, die neben der regionalen und nationalen Bedeutung auch weltweite Vergleiche zulassen. Das WHO-MONICA-Projekt hat deshalb über die so genannten ökologischen Analysen hinaus dazu beigetragen, dass in vielen Regionen der Welt die Grundlagen für Herz-Kreislauf-Epidemiologie und -Prävention gelegt werden konnten. In vielen MONICAZentren sind auf diese Weise auch klassische Kohortenstudien von Symptomen bzw. 24 Stunden bis 28 Tage nach Beginn von Symptomen - Altersgruppe 35-64 Jahre [11].

entstanden, die auf vielen Gebieten der Herz-Kreislauf-Forschung wichtige und innovative Beiträge geleistet haben und zum besseren Verständnis der Ätiologie dieser Krankheitsgruppe beigetragen haben. Für die Region Augsburg sind Beiträge zu folgenden Gebieten besonders erwähnenswert: Ätiologie der Herzinsuffizienz, Ernährung und KHK, Alkohol und KHK, die Bedeutung von Fibrinogen, C-reaktivem Protein und Blutviskosität für KHK und die besondere Bedeutung von Diabetes und Hypertonie für die Herz-Kreislauf-Mortalität und -Morbidität. Darüber hinaus werden die Datenbasen des MONICA-Projektes Augsburg sowohl für Fragen der genetischen Epidemiologie wie auch der Versorgungsforschung genutzt.

Neben der Sieben-Länder-Studie und der Framingham-Studie kommt dem WHO-MONICA-Projekt die größte Bedeutung für die Weiterentwicklung der Herz-Kreislauf-Epidemiologie und -Prävention in der Welt zu [5]. In Deutschland hat das MONICAProjekt Augsburg in den 80er- und 90er-Jahren sehr wesentlich zum Aufbau und zur Etablierung einer international konkurrenzfähigen Herz-Kreislauf-Epidemiologie beigetragen. Allein aus dem MONICA-Projekt Augsburg sind über 200 Artikel in hochkarätigen wissenschaftlichen Zeitschriften publiziert worden.

\section{Literatur}

${ }^{1}$ Havlik RJ, Feinleib M (eds). Proceedings of the Conference on the decline in coronary heart disease mortality, Oct 24-25, 1978 at National Heart, Lung, and Blood Institute, Washington DC, US Department of Health, Education, and Welfare, 1979 (NIH publication no 79-1610) 
${ }^{2}$ The WHO MONICA Principal Investigators. Monitoring Trends and Determinants in Cardiovascular Disease. A Major International Collaboration. J Clin Epidemiol 1988; 41: 105-114

${ }^{3}$ Tunstall-Pedoe H, Kuulasmaa K, Mähönen M et al. Contribution of trends in survival and coronary-event rates to changes in coronary heart disease mortality: 10-year results from 37 WHO MONICA Project populations. Lancet 1999; 353: $1547-1557$

${ }^{4}$ Alpert J. Commentary. Coronary heart disease: where have we been and where are we going? Lancet 1999; 353: 1540

${ }^{5}$ Tunstall-Pedoe H (ed). MONICA Monograph and Multimedia, Sourcebook. World's largest study of heart disease, stroke, risk factors, and population trends 1979-2002. Geneva: World Health Organization, 2003

${ }^{6}$ Kuulasmaa K, Tunstall-Pedoe H, Dobson A et al. Estimation of contribution of changes in classic risk factors to trends in coronary-event rates across the WHO MONICA Project populations. Lancet 2000; 355: 675-687

${ }^{7}$ Magnus P, Beaglehole R. The real contribution of the major risk factors to the coronary epidemics. Time to end the „only-50\%“ myth. Arch Intern Med 2001; 161: 2657-2660

${ }^{8}$ Keil U, Liese AD, Hense HW et al. Classical risk factors and their impact on incident non-fatal and fatal myocardial infarction and all-cause mortality in southern Germany. Results from the MONICA Augsburg cohort study 1984 - 1992. Eur Heart J 1998; 19: 1197-1207

${ }^{9}$ Tunstall-Pedoe H, Vanuzzo D, Hobbs M et al. Estimation of contribution of changes in coronary care to improving survival, event rates, and coronary heart disease mortality across the WHO MONICA Project populations. Lancet 2000; 355: 688-700

10 Keil U, Filipiak B, Döring A et al. Mortalität an Herz-Kreislauf-Krankheiten von 1985 bis 1990. Abnahme bei Männern - Zunahme bei Frauen. Therapiewoche 1993; 43: 1658-1664

${ }^{11}$ Chambless L, Keil U, Dobson A et al. for the WHO MONICA Project. Population versus clinical view of case fatality from acute coronary heart disease: results from the WHO MONICA Project 1985-1990. Circulation 1997; 96: 3849-3859 